\title{
Draft genomes of four enterotoxigenic Escherichia coli (ETEC) clinical isolates from China and Bangladesh
}

Fei Liu ${ }^{1,2}$, Xi Yang ${ }^{1}$, Zhiyun Wang ${ }^{1}$, Matilda Nicklasson ${ }^{3}$, Firdausi Qadri ${ }^{4}$, Yong Yi ${ }^{5}$, Yuying Zhu ${ }^{1}$, Na Lv' ${ }^{1}$, Jing Li ${ }^{1}$, Ruifen Zhang ${ }^{1}$, Huijuan Guo ${ }^{1}$, Baoli Zhu ${ }^{1,2}$, Åsa Sjöling ${ }^{3,6^{*}}$ and Yongfei $\mathrm{Hu}^{1,2^{*}}$

\begin{abstract}
Background: Enterotoxigenic Escherichia coli (ETEC) is an important pathogen that causes childhood and travelers' diarrhea. Here, we present the draft genomes of four ETEC isolates recovered from stool specimens of patients with diarrhea in Beijing, China and Dhaka, Bangladesh, respectively.

Results: We obtained the draft genomes of ETEC strains CE516 and CE549 isolated in China, and E1777 and E2265 isolated in Bangladesh with a length of $5.1 \mathrm{Mbp}, 4.9 \mathrm{Mbp}, 5.1 \mathrm{Mbp}$, and 5.0 Mbp, respectively. Phylogenetic analysis indicated that the four strains grouped with the classical Escherichia coli phylogenetic groups A and B1 and three of them including a multi drug-resistant Chinese isolate (CE549) belonged to two major ETEC lineages distributed globally. The heat stable toxin (ST) structural gene (estA) was present in all strains except in strain CE516, and the heat labile toxin (LT) operon (eltAB) was present in all four genomes. Moreover, different resistance gene profiles were found between the ETEC strains.

Conclusions: The draft genomes of the two isolates CE516 and CE549 represent the first genomes of ETEC reported from China. Though we revealed that ETEC is uncommon in Beijing, China, however, when it does occur, multi-drug resistance and ESBL positive isolates might pose a specific public health risk. Furthermore, this study advances our understanding of prevalence and antibiotic resistance of ETEC in China and adds to the number of sequenced strains from Bangladesh.
\end{abstract}

Keywords: ETEC, Virulence factors, Antibiotic resistance

\section{Background}

ETEC infections are an important cause of childhood diarrhea resulting in significant morbidity and mortality, primarily among children aged $<5$ years living in developing countries [1] as well as travelers visiting these countries [2]. ETEC is characterized by the presence of the heat-labile toxin (LT) and/or the heat-stable toxin (ST), both of which are plasmid encoded [3]. The presence of virulence factors such as enterotoxins and colonization factors differentiate ETEC from other categories of diarrheagenic E. coli. [4]. Colonization factors

\footnotetext{
* Correspondence: asa.sjoling@ki.se; huyf@im.ac.cn

${ }^{3}$ Department of Microbiology and Immunology, Institute of Biomedicine, University of Gothenburg, Box 435, 40530 Göteborg, Sweden

'CAS Key Laboratory of Pathogenic Microbiology \& Immunology, Institute of Microbiology, Chinese Academy of Sciences, Beijing 100101, PR China Full list of author information is available at the end of the article
}

(CFs) enable ETEC bacteria to adhere to the intestinal epithelium [5]. At present more than 25 different CFs have been identified [5]. In addition to the CFs, other putative factors involved in ETEC pathogenesis were also identified, such as EtpA and EatA. EtpA can act as a bridge between the bacterial flagella and host epithelial cells [6] and EatA is a protein of the serine protease autotransporters of the Enterobacteriaceae (SPATE) family [7].

For a long time E. coli $\mathrm{H} 10407$ and E24377A were the only two ETEC strains infecting humans that have their genomes completely sequenced together with a draft genome of ETEC strain B7A [8,9]. Recently whole genome sequences of additional draft genomes were published [10]. A comprehensive analysis of 362 ETEC genomes from strains isolated globally over three decades identified that ETEC distribute into several conserved monophyletic 
lineages that have distrubuted globally [11] . In this study we analysed four additional ETEC strains with the aim to compare additional ETEC isolated in China and Bangladesh with the global collection and to better understand the dissemination of the pathogen. We also included two additional Bangladeshi strains to increase the number of sequenced genomes from Bangladeshi ETEC strains.

\section{Methods}

\section{Strain selection}

To assess the frequency of ETEC in Beijing, China, we investigated patients presenting with acute watery diarrhea at four hospitals between 2010 and 2011. This research was approved by the Research Ethics Committee of the Institute of Microbiology, Chinese Academy of Sciences. ETEC isolates were recovered after streaking diarrheal samples on to MacConkey agar followed by PCR confirmation for ETEC-specific enterotoxins [12]. In total, 880 cases were enrolled and tested for ETEC but ETEC was only recovered from three cases $(0.3 \%)$. The two ETEC isolates CE516 and CE549 from China were recovered from stool of patients that tested negative for Vibrio cholerae, Shigella spp and Salmonella spp. CE549 expressed the heat-labile enterotoxin (LT) and the human heat-stabile enterotoxin (STh) in combination with CFs CS2, CS3 and CS21; CE516 expressed LT and CS6, CS8. Antimicrobial susceptibility was determined using the VITEK 2 Gram Negative Susceptibility Test Cards AST-GN04 and AST-GN 13 (Biomerieux, Marcy l'Etoile France). CE549 was resistant to 14 of the 22 antibiotics tested (cefuroxime axetil, sulfamethoxazole, ampicillin, tobramycin, ceftriaxone, aztreonam, piperacillin, cefuroxime, cefazolin, ceftazidime, cefepime, levofloxacin, gentamicin, ciprofloxacin, and extended spectrum beta lacatamase (ESBL) positive), while CE516 showed sensitive to all 22 antibiotics and was ESBL negative.

The two ETEC isolates E1777 and E2265 were collected from adult Bangladeshi patients that sought medical attention for severe diarrhea in hospital facilities in April 2005 and March 2006 during the bi-annual ETEC epidemic peaks in Dhaka, Bangladesh [13]. Stool samples were confirmed to be negative for Vibrio cholerae, Shigella ssp and Salmonella ssp. MacConkey agar plates were used for identification of lactose fermenting $E$. coli like colonies selection followed by PCR confirmation for ETEC [12]. The strains were further characterized by immunodiagnostic methods for toxins and colonization factors [12]. Both isolates expressed the common virulence factor combination of the enterotoxins heat labile toxin LT and heat stable toxin STh and the CFs CS5 and CS6.

\section{Genome sequencing, assembly and annotation}

DNA was extracted from bacterial cells cultured in Luria broth (LB) medium using the DNA Tissue and Blood kit (Qiagen, Duesseldorf, Germany). Genome sequencing work was carried out at the Microbial Genome Research Center, Institute of Microbiology, Chinese Academy of Sciences, Beijing. The genome sequences of each ETEC isolate were generated using paired-end libraries with $350 \sim 400$ bp inserts on an Illumina GAIIX (Illumina, San Diego, CA, USA). The detailed methods for genome assembly were described in another paper [14]. Genome sequences were annotated by using Subsystem Technology (RAST) [15]. The functions of predicted protein-coding genes were then annotated through comparisons with the databases of NCBI-NR, and COG. To search the antibiotic resistance genes, the protein-coding sequences were aligned against Antibiotic Resistance Database

Table 1 Reference strains used for this study

\begin{tabular}{|c|c|c|c|c|c|c|}
\hline Strain & GenBank BioSample & Accession number & Collection date & Isolation source & Genome size (bp) & GC content \\
\hline B7A & SAMN02435852 & NZ_AAJT02000001.1 & $\backslash$ & $\backslash$ & $5,300,242$ & $50.7 \%$ \\
\hline E24377A & SAMN02604038 & NC_009786.1 & $\backslash$ & $\backslash$ & $5,249,288$ & $50.6 \%$ \\
\hline $\mathrm{H} 10407$ & SAMEA2272237 & NC_017633.1 & prior to 1973 & $\backslash$ & $5,325,888$ & $50.7 \%$ \\
\hline$|A| 39$ & SAMEA3138234 & NC_011750.1 & $\backslash$ & $\backslash$ & $5,132,068$ & $50.6 \%$ \\
\hline O127 H6 E2348/69 & SAMEA1705959 & NC_011601.1 & 1969 & $\backslash$ & $5,069,678$ & $50.5 \%$ \\
\hline O157 H7 EC4115 & SAMN02603441 & NC_011350.1 & $\backslash$ & $\backslash$ & $5,704,171$ & $50.4 \%$ \\
\hline O157 H7 EDL933 & SAMN02604092 & NC_002655.2 & $\backslash$ & $\backslash$ & $5,620,522$ & $50.4 \%$ \\
\hline O157 H7 TW14359 & SAMN02604255 & NC_013008.1 & $\backslash$ & $\backslash$ & $5,622,737$ & $50.5 \%$ \\
\hline 0157 H7 Sakai & SAMN01911278 & NC_002127.1 & 1996 & Human feces & $5,594,477$ & $50.5 \%$ \\
\hline SMS-3-5 & SAMN02604066 & NC_010485.1 & $\backslash$ & $\backslash$ & $5,215,377$ & $50.5 \%$ \\
\hline TW10598 & SAMN02436015 & NZ_AELA01000001.1 & $\backslash$ & $\backslash$ & $5,243,318$ & $50.6 \%$ \\
\hline TW10722 & SAMN02435971 & NZ_AELB01000001.1 & $\backslash$ & $\backslash$ & $5,689,893$ & $50.5 \%$ \\
\hline TW10828 & SAMN02435898 & NZ_AELC01000001.1 & $\backslash$ & $\backslash$ & $5,280,267$ & $50.6 \%$ \\
\hline
\end{tabular}


Table 2 Genomic characteristics of the 4 ETEC genomes

\begin{tabular}{|c|c|c|c|c|c|c|c|c|c|}
\hline Sample name & Country & MLST & Colonization factors & ST & LT & Read length (bp) & Genome coverage & GC content & Scaffold numbe \\
\hline CE516 & China & 1490 & CS6, CS8 & - & + & 101 & $300 x$ & $50.5 \%$ & 99 \\
\hline CE549 & China & 4 & $\mathrm{CS} 2, \mathrm{CS} 3, \mathrm{CS} 21$ & + & + & 101 & $300 x$ & $50.6 \%$ & 137 \\
\hline E1777 & Bangladeshi & 443 & CS5, CS6 & + & + & 101 & $200 x$ & $50.4 \%$ & 150 \\
\hline E2265 & Bangladeshi & 443 & CS5, CS6 & + & + & 101 & $200 x$ & $50.3 \%$ & 142 \\
\hline
\end{tabular}

(ARDB) [16], using similarity thresholds as recommended in ARDB.

\section{Multiple locus sequence typing (MLST)}

We used MLST system including the following seven housekeeping genes: $a d k, f u m C, \operatorname{gyr} B$, icd, $m d h$, purA, and $\operatorname{rec} A$ [17], which were extracted from draft genome sequences and were compared to allele profiles in the MLST database (http://mlst.warwick.ac.uk/mlst/ dbs/Ecoli/documents/primersColi_html).

\section{Comparative genomics}

For comparative genomic analysis, genome sequences of 13 previously reported isolates including Escherichia coli B7A (GenBank accession number NZ_AAJT02000001.1), E24377A (NC_009786.1), H10407 (NC_017633.1), IAI39 (NC_011750.1), O127 H6 E2348 69 (NC_011601.1), O157 H7 EC4115 (NC_011350.1), O157 H7 EDL933 (NC_002655.2), O157 H7 TW14359 (NC_013008.1), O157 H7 Sakai (NC_002127.1), SMS 35 (NC_010485.1), TW10598 (NZ_AELA01000001.1), TW10722 (NZ_AELB01000001.1),

Table 3 Comparisons of subsystem features among the 4 ETEC genomes

\begin{tabular}{|c|c|c|c|c|}
\hline \multirow[t]{2}{*}{ Subsystem features } & \multicolumn{4}{|c|}{ Number of CDS present in ETEC strains } \\
\hline & CE516 & CE549 & E1777 & E2265 \\
\hline Amino Acids and Derivatives & 400 & 391 & 392 & 395 \\
\hline Carbohydrates & 781 & 756 & 752 & 754 \\
\hline Cell Division and Cell Cycle & 39 & 40 & 38 & 37 \\
\hline Cell Wall and Capsule & 267 & 273 & 272 & 273 \\
\hline Cofactors, Vitamins, Prosthetic Groups, Pigments & 285 & 285 & 287 & 284 \\
\hline DNA Metabolism & 129 & 147 & 153 & 134 \\
\hline Dormancy and Sporulation & 4 & 5 & 5 & 5 \\
\hline Fatty Acids, Lipids, and Isoprenoids & 142 & 131 & 132 & 131 \\
\hline Iron acquisition and metabolism & 22 & 22 & 22 & 22 \\
\hline Membrane Transport & 291 & 190 & 268 & 270 \\
\hline Metabolism of Aromatic Compounds & 44 & 5 & 30 & 30 \\
\hline Miscellaneous & 67 & 63 & 66 & 64 \\
\hline Motility and Chemotaxis & 80 & 130 & 80 & 80 \\
\hline Nitrogen Metabolism & 77 & 75 & 77 & 77 \\
\hline Nucleosides and Nucleotides & 146 & 150 & 147 & 144 \\
\hline Phages, Prophages, Transposable elements, Plasmids & 130 & 32 & 160 & 146 \\
\hline Phosphorus Metabolism & 53 & 53 & 53 & 53 \\
\hline Photosynthesis & 0 & 0 & 0 & 0 \\
\hline Potassium metabolism & 29 & 29 & 28 & 30 \\
\hline Protein Metabolism & 299 & 290 & 298 & 300 \\
\hline Regulation and Cell signaling & 160 & 156 & 160 & 163 \\
\hline Respiration & 192 & 190 & 194 & 192 \\
\hline RNA Metabolism & 248 & 251 & 250 & 250 \\
\hline Secondary Metabolism & 27 & 26 & 26 & 26 \\
\hline Stress Response & 184 & 181 & 186 & 184 \\
\hline Sulfur Metabolism & 59 & 54 & 56 & 56 \\
\hline Virulence, Disease and Defense & 109 & 108 & 110 & 130 \\
\hline
\end{tabular}


and TW10828 (NZ_AELC01000001.1) were downloaded from the NCBI website (Table 1). Multiple sequence alignments of Escherichia coli genomes were performed with Mugsy [18]. The trees were constructed based on core SNPs (single nucleotide polymorphisms) from whole genome alignment by using the maximum-likelihood method in Phylogeny Inference Package (http://evolution. genetics.washington.edu/phylip.html). The map of ORF comparisons among E. coli genomes was constructed using Circos [19].

\section{Quality assurance}

The genomic DNA was isolated from pure bacterial isolate and was further confirmed with 16S rRNA gene sequencing. Bioinformatic assessment of potential contamination of the genomic library by allochthonous microorganisms was done using PGAAP and RAST annotation system.

\section{Initial findings}

\section{Genome characteristics}

Through genome assembly, we obtained 99 scaffolds of 5,068,634 bp for CE516, 137 scaffolds of 4,859,890 bp for CE549, 150 scaffolds of 5,117,746 bp for E1777, and 142 scaffolds of 4,946,932 bp for E2265 (Table 2). RAST annotation of the whole genome indicated the presence of 611, 590, 605, and 605 SEED subsystems in CE516, CE549, E1777, and E2265, respectively. Table 3 shows the comparison of genomic features of the four sequenced ETEC genomes.

\section{Phylogenetic analysis}

A maximum-likelihood tree of the sequenced 4 genomes and 13 publicly available Escherichia coli complete genomes which represent the classical phylogenetic groups (A, B1, B2, D, and E) were created based on core SNPs from whole genome alignment (Figure 1). The sequenced strains in this study grouped with the classical Escherichia coli phylogenetic groups A and B1. Specifically, strains CE549, H10407 and TW10598 which belong to group A were grouped together, while other sequenced strains which belong to group B1 as well as the previously sequenced strains formed a clade. Strains CE549 and TW10598 are closely related to each other, while strains E1777 and E2265 are closely related to each other. MLST analysis was used to compare the strains to a global collection of ETEC [11]. Three strains were found to belong to the major lineages described in ETEC [11]. Strains E1777 and E2265 belong to the global lineage L5 which express LT STh CS5 + CS5, while strain $\mathrm{CE} 156$, the multi drug-resistant isolate belongs to the conserved ETEC lineage L2 that is distributed globally [11]. The Chinese strain CS516 belonged to a MLST type previously identified in Bangladeshi and Egyptian ETEC strains [11].

\section{Genomic variants among ETEC strains}

We compared proteins from the 4 draft genomes and 6 references within groups A and B1 with that from H10407 using BLASTP and revealed many large variable regions (VR1 to VR10) (Figure 2). Among these VRs, VR3 and VR10 (regions of 5,072 to 5,121 kb) were predicted to be prophage loci which were highly variable among all strains. Interestingly, all strains within group B1 lack VR7 gene cluster encoding general secretory pathway associated genes. In addition, region 2,405 to 2,414 $\mathrm{kb}$ adjacent to VR4, which encoded ribitol metabolism related genes, was presented within group A but not detected within group B1.

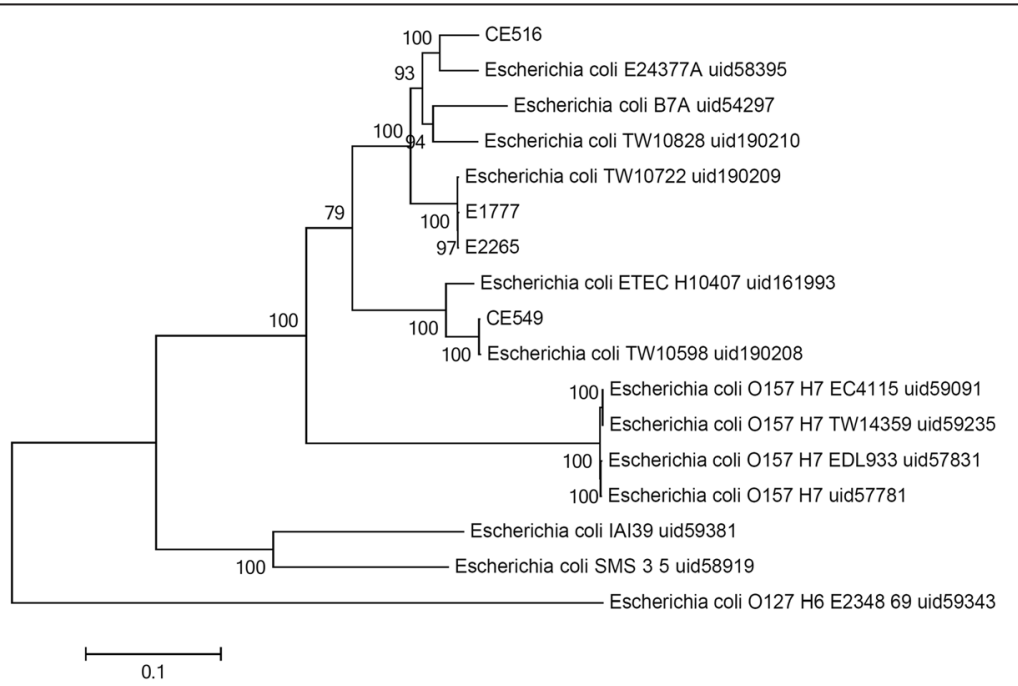

Figure 1 Phylogenetic relationships of $E$. coli strains based on SNPs from whole genome sequences. The trees were constructed by the maximum-likelihood method. Scale bar indicates nucleotides substitutions per site. 


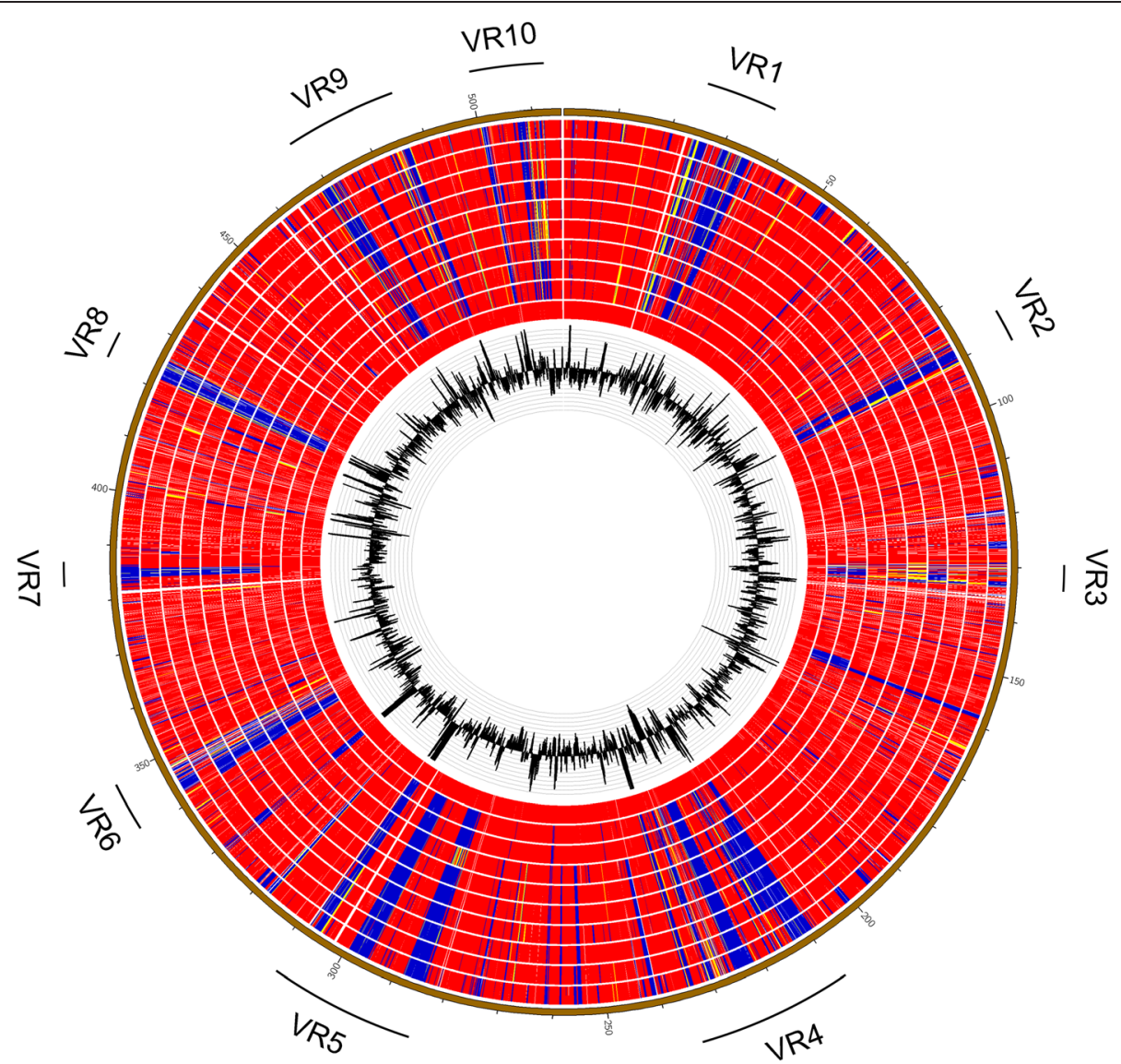

Figure 2 ORF comparisons of E. coli genomes. Proteins from the 4 genomes and 6 references within groups A and B1 were aligned using H10407 as a reference. Track shows a plot of $\mathrm{G}+\mathrm{C}$ contents. Circles from inside to outside are the BLASTP percent identities of H10407 against ORFs of H10407, TW10598, CE549, TW10722, E1777, E2265, E24377A, CE516, TW10828, B7A. Red is 90-100\% identity, yellow is 60-89\% identity, blue is $0-59 \%$ identity.

\section{Virulence factors}

The strains were analyzed for presence of known ETEC virulence factors. Strains E1777, E2265, and CE549 contained both LT and ST genes (Table 4). The ST structural gene (estA) was present in all strains except in strain CE516, while the LT structural gene (eltA) was present in all four genomes. In addition, genes clyA (cytolysin), eatA (serine protease autotransporter), and ecpA (pilus subunit) were also present in all of the 4 ETEC strains, but genes leoA (accessory protein for LT secretion), tibA (autotransporter), and tia (surface protein) were absent in all genomes. Only CE549 contained the complete $\sim 14 \mathrm{~kb}$ operon encoding longus known as a type IV pilus [20]. The etpA gene, which mediates adhesion between ETEC flagella and host cells [6], was present only in CE549 but absent in other strains. These specific virulence factors present in CE549 may increase its virulence in humans, but their functional effects remain to be further determined.
Table 4 Virulence factors present or absent in the 4 ETEC genomes

\begin{tabular}{lllll}
\hline Virulence factor & CE516 & CE549 & E1777 & E2265 \\
\hline clyA & 1 & 1 & 1 & 1 \\
eatA & 1 & 1 & 1 & 1 \\
ecpA & 1 & 1 & 1 & 1 \\
eltA & 1 & 1 & 1 & 1 \\
estA & 0 & 1 & 1 & 1 \\
etpA & 0 & 1 & 0 & 0 \\
fimH & 1 & 1 & 1 & 1 \\
leoA & 0 & 0 & 0 & 0 \\
IngA & 0 & 1 & 0 & 0 \\
tia & 0 & 0 & 0 & 0 \\
tibA & 0 & 0 & 0 & 0 \\
\hline
\end{tabular}

" 1 " and " 0 " denotes the presence and absence of the corresponding virulence factors. 


\section{Antibiotic resistance genes}

We compared all the protein-coding genes from the 4 ETEC strains with known antibiotic resistance genes [16] and found many kinds of antibiotic resistance genes, such as macrolide, tetracycline, fosmidomycin and polymyxin resistance genes (Table 5), most of which were annotated as Multidrug resistance efflux pump. Interestingly, strain CE549 has two tetracycline resistance genes that were not identified in the other 3 isolates. In addition, different resistance genes profiles were found between ETEC strains from different countries. For instance, the resistant type EmrE was only identified in the two strains isolated from China.

\section{Future directions}

This study analyzed the prevalence of ETEC in Beijing, China and it was found that ETEC is not common. However the results reveal for the first time to our knowledge that a strain that belong to the globally distributed ETEC lineage L2 is multi resistant. This might have important implications for transmission of multi resistant ETEC strains as well as treatment of ETEC diarrhea and needs to be further addressed. The Chinese genomes presented here together with the two novel Bangladeshi ETEC genomes, will be valuable for future comparative genomic analysis of ETEC and will aid in molecular characterization of this important diarrheal pathogen.

Table 5 Putative antibiotic resistance genes in the 4 ETEC strains determined using the antibiotic resistance genes database

\begin{tabular}{|c|c|c|c|c|c|c|}
\hline Resistance type & Description & Resistance profile & CE516 & CE549 & E1777 & E2265 \\
\hline acrA & Multidrug resistance efflux pump. & aminoglycoside, glycylcycline, & * & $*$ & * & * \\
\hline $\mathrm{acrB}$ & & Deta_lactam, macrollde, acritavin & $*, *$ & $*^{*}{ }^{*}$ & $*{ }^{*},{ }^{*}$ & $*{ }^{*}, *$ \\
\hline $\operatorname{arnA}$ & $\begin{array}{l}\text { The modified arabinose is attached to lipid A } \\
\text { and is required for resistance to polymyxin } \\
\text { and cationic antimicrobial peptides. }\end{array}$ & polymyxin & * & * & * & * \\
\hline bacA & $\begin{array}{l}\text { Undecaprenyl pyrophosphate phosphatase, } \\
\text { which consists in the sequestration of } \\
\text { Undecaprenyl pyrophosphate. }\end{array}$ & bacitracin & * & * & * & * \\
\hline bcr & & & * & * & * & * \\
\hline bl1_ec & Class $C$ beta-lactamase. & cephalosporin & * & * & * & * \\
\hline emrD & Multidrug resistance efflux pump. & & * & * & * & * \\
\hline emrE & & aminoglycoside & $*{ }^{*}, *$ & * & & \\
\hline $\mathrm{ksgA}$ & $\begin{array}{l}\text { Its inactivation leads to kasugamycin } \\
\text { resistance. }\end{array}$ & kasugamycin & * & * & * & * \\
\hline $\operatorname{macB}$ & Macrolide-specific efflux system. & macrolide & * & * & * & * \\
\hline mdfA & & & * & * & * & * \\
\hline mdtE & Multidrug resistance efflux pump. & doxorubicin, erythromycin & * & * & * & * \\
\hline mdtF & & & * & * & * & * \\
\hline mdtG & Multidrug resistance efflux pump. & deoxycholate, fosfomycin & * & * & * & * \\
\hline $\mathrm{mdtH}$ & & & * & * & $*, *$ & $*^{*}$, \\
\hline mdtK & & enoxacin, norfloxacin & * & * & * & * \\
\hline mdtL & & chloramphenicol & * & * & * & * \\
\hline mdtM & & chloramphenicol, acriflavine, norfloxacin & * & * & * & * \\
\hline $\mathrm{mdtN}$ & Multidrug resistance efflux pump. & t_chloride, acriflavine, puromycin & * & * & * & * \\
\hline mdtO & & & * & * & * & * \\
\hline mdtP & & & * & * & * & * \\
\hline $\operatorname{ros} B$ & $\begin{array}{l}\text { Efflux pump/potassium antiporter system. } \\
\text { RosB: Potassium antiporter. }\end{array}$ & fosmidomycin & * & * & * & * \\
\hline tetC & $\begin{array}{l}\text { Major facilitator superfamily transporter, } \\
\text { tetracycline efflux pump. }\end{array}$ & tetracycline & & $*^{*}, *$ & & \\
\hline tolc & Multidrug resistance efflux pump. & $\begin{array}{l}\text { aminoglycoside, glycylcycline, } \\
\text { beta_lactam, macrolide, acriflavin }\end{array}$ & * & * & * & * \\
\hline
\end{tabular}

"**" means one homolog of the antibiotic resistance gene is found. 


\section{Availability of supporting data}

The genome sequences of ETEC strains CE516, CE549, E1777 and E2265 reported in this paper have been deposited in the GenBank under accession numbers JTGM00000000, JTGK00000000, JTHI00000000 and JUBB00000000, respectively.

\section{Competing interests}

The authors declare that they have no competing interests.

\section{Authors' contributions}

FL interpreted the sequencing data and prepared the manuscript. NL and YYZ generated the sequencing data. BLZ, YFH and Ås participated in all discussions of data analysis and manuscript revisions. ZYW, MN and $\AA S$ analyzed the stool samples. FL, XY, ZYW, FQ, YY, JL, RFZ, HJG, YFH, ÅS and BLZ were involved in overall experimental design. All authors have read the manuscript and approved.

\section{Acknowledgements}

This work was supported by National Natural Science Foundation of China (grant 31270168 and 81401701), the National Basic Research Program of China (973 Program: grant 200CB504800), the Beijing Municipal Natural Science Foundation (5152019), the Swedish Research Council (grant no 521-2011-3435) and the Swedish Research Links (348-2011-7292) to ÅS and BLZ

\section{Author details}

'CAS Key Laboratory of Pathogenic Microbiology \& Immunology, Institute of Microbiology, Chinese Academy of Sciences, Beijing 100101, PR China. ${ }^{2}$ Collaborative Innovation Center for Diagnosis and Treatment of Infectious Diseases, The First Affiliated Hospital, College of Medicine, Zhejiang University, Hangzhou 310006, China. ${ }^{3}$ Department of Microbiology and Immunology, Institute of Biomedicine, University of Gothenburg, Box 435, 40530 Göteborg, Sweden. ${ }^{4}$ International Centre for Diarrhoeal Disease Research, Bangladesh, GPO Box 128, Dhaka 1000, Bangladesh. ${ }^{5}$ Clinical Diagnostic Center, 306nd Hospital of the People's Liberation Army, Beijing 100101, PR China. ${ }^{6}$ Department of Microbiology, Tumor and Cell Biology, Karolinska Institutet, Box 280, 17177 Stockholm, Sweden.

Received: 19 December 2014 Accepted: 19 March 2015

Published online: 08 April 2015

\section{References}

1. Qadri F, Svennerholm AM, Faruque AS, Sack RB. Enterotoxigenic Escherichia coli in developing countries: epidemiology, microbiology, clinical features, treatment, and prevention. Clin Microbiol Rev. 2005:18:465-83.

2. Black RE. Epidemiology of travelers' diarrhea and relative importance of various pathogens. Rev Infect Dis. 1990;12 Suppl 1:S73-9.

3. Fleckenstein JM, Hardwidge PR, Munson GP, Rasko DA, Sommerfelt H, Steinsland H. Molecular mechanisms of enterotoxigenic Escherichia coli infection. Microbes Infect. 2010;12:89-98.

4. Nataro JP, Kaper JB. Diarrheagenic Escherichia coli. Clin Microbiol Rev. 1998:11:142-201.

5. Gaastra W, Svennerholm AM. Colonization factors of human enterotoxigenic Escherichia coli (ETEC). Trends Microbiol. 1996:4:444-52.

6. Roy K, Hilliard GM, Hamilton DJ, Luo J, Ostmann MM, Fleckenstein JM. Enterotoxigenic Escherichia coli EtpA mediates adhesion between flagella and host cells. Nature. 2009;457:594-8.

7. Henderson IR, Cappello R, Nataro JP. Autotransporter proteins, evolution and redefining protein secretion. Trends Microbiol. 2000;8:529-32.

8. Crossman LC, Chaudhuri RR, Beatson SA, Wells TJ, Desvaux M, Cunningham $\mathrm{AF}$, et al. A commensal gone bad: complete genome sequence of the prototypical enterotoxigenic Escherichia coli strain H10407. J Bacteriol. 2010;192:5822-31

9. Rasko DA, Rosovitz MJ, Myers GS, Mongodin EF, Fricke WF, Gajer P, et al. The pangenome structure of Escherichia coli: comparative genomic analysis of E. coli commensal and pathogenic isolates. J Bacteriol. 2008;190:6881-93.

10. Sahl JW, Steinsland H, Redman JC, Angiuoli SV, Nataro JP, Sommerfelt $\mathrm{H}$ et al. A comparative genomic analysis of diverse clonal types of enterotoxigenic Escherichia coli reveals pathovarspecific conservation. Infect Immun. 2011; 79:950-60
11. von Mentzer A, Connor TR, Wieler LH, Semmler T, Iguchi A, Thomson NR, et al. Identification of enterotoxigenic Escherichia coli (ETEC) clades with long-term global distribution. Nat Genet. 2014;46:1321-6.

12. Sjoling A, Wiklund G, Savarino SJ, Cohen DI, Svennerholm AM. Comparative analyses of phenotypic and genotypic methods for detection of enterotoxigenic Escherichia coli toxins and colonization factors. J Clin Microbiol. 2007;45:3295-301.

13. Nicklasson M, Sjoling A, von Mentzer A, Qadri F, Svennerholm AM Expression of colonization factor CS5 of enterotoxigenic Escherichia coli (ETEC) is enhanced in vivo and by the bile component Na glycocholate hydrate. PLoS One. 2012;7:e35827.

14. Liu F, Hu Y, Wang Q, Li HM, Gao GF, Liu CH, et al. Comparative genomic analysis of Mycobacterium tuberculosis clinical isolates. BMC Genomics. 2014;15:469

15. Aziz RK, Bartels D, Best AA, DeJongh M, Disz T, Edwards RA, et al. The RAST Server: rapid annotations using subsystems technology. BMC Genomics. 2008:9:75.

16. Liu B, Pop M. ARDB-Antibiotic Resistance Genes Database. Nucleic Acids Res. 2009:37:D443-7.

17. Wirth T, Falush D, Lan R, Colles F, Mensa P, Wieler LH, et al. Sex and virulence in Escherichia coli: an evolutionary perspective. Mol Microbiol. 2006:60:1136-51.

18. Angiuoli SV, Salzberg SL. Mugsy: fast multiple alignment of closely related whole genomes. Bioinformatics. 2010;27:334-42.

19. Krzywinski M, Schein J, Birol I, Connors J, Gascoyne R, Horsman D, et al. Circos: an information aesthetic for comparative genomics. Genome Res. 2009;19:1639-1645.

20. Gomez-Duarte OG, Chattopadhyay S, Weissman SJ, Giron JA, Kaper JB, Sokurenko EV. Genetic diversity of the gene cluster encoding longus, a type IV pilus of enterotoxigenic Escherichia coli. J Bacteriol. 2007;189:9145-9.

\section{Submit your next manuscript to BioMed Central and take full advantage of:}

- Convenient online submission

- Thorough peer review

- No space constraints or color figure charges

- Immediate publication on acceptance

- Inclusion in PubMed, CAS, Scopus and Google Scholar

- Research which is freely available for redistribution 